\title{
Velhos indicadores para novos problemas: a relação entre saneamento e saúde
}

\author{
Marco Antonio Ratzsch Andreazzi, ${ }^{1}$ Christovam Barcellos ${ }^{2}$ \\ e Sandra Hacon ${ }^{3}$
}

Como citar Andreazzi MAR, Barcellos C, Hacon S. Velhos indicadores para novos problemas: a relação entre saneamento e saúde. Rev Panam Salud Publica. 2007:22(3):000-00.

RESUMO Objetivo. Revisar a literatura publicada entre 1995 e 2004 acerca da relação entre saneamento e saúde e levantar as principais variáveis analisadas e as doenças ou agravos usados como marcadores de efeito ou de risco ambiental.

Método. Realizou-se uma busca nas bases de dados Medline, SciELO e LILACS utilizando os termos "sanitation" $e$ "health" $e$ "indicator" $e$ "water" (saneamento, saúde, indicador $e$ água). Foram identificados 103 artigos, sendo que 17 foram considerados pertinentes à análise proposta. Foram identificados, para cada artigo, o desenho do estudo e as variáveis de saúde e de saneamento avaliadas.

Resultados. O desenho ecológico foi o mais freqüente, tendo sido utilizado em sete dos 17 estudos. A maioria dos artigos enfocava a diarréia como variável de saúde (10 estudos) e a qualidade da água como variável de saneamento (10 estudos). Em 15 estudos foi observada uma associação positiva entre a variável de saúde e a variável de saneamento investigadas.

Conclusões. Ainda restam muitas lacunas no entendimento do saneamento e de seu papel, principalmente nos grandes centros urbanos, onde a dependência dos sistemas de água e esgotamento sanitário é muito grande, não existindo, na maioria das vezes, fontes alternativas de abastecimento. Os estudos ecológicos construídos a partir de dados secundários e os inquéritos específicos têm se destacado como uma boa opção para análises envolvendo as relações entre condições de saneamento e saúde.

Palavras-chave Desenvolvimento local, indicadores básicos de saúde, monitoramento ambiental, saneamento, saúde ambiental, Brasil.

1 Instituto Brasileiro de Geografia e Estatística (IBGE) e Fundação Oswaldo Cruz (FIOCRUZ), Escola Nacional de Saúde Pública (ENSP). Enviar correspondência para este autor no seguinte endereço: IBGE, Avenida Chile 500, $8^{\circ}$ andar, CEP 20031-170, Rio de Janeiro, RJ, Brasil. Telefone: +5521-2142.0373/21-2142.0033; e-mail: mandreazzi@ terra.com.br

2 Fundação Oswaldo Cruz (FIOCRUZ), Centro de Informação Científica e Tecnológica (CICT), Departamento de Informação em Saúde, Rio de Janeiro, RJ, Brasil. E-mail: xris@cict.fiocruz.br

3 Fundação Oswaldo Cruz (FIOCRUZ), Escola Nacional de Saúde Pública Sergio Arouca (ENSP), Departamento de Endemias Samuel Pessoa, Rio de Janeiro, RJ, Brasil.
As concepções sobre a relação entre saneamento e saúde têm se desenvolvido junto com a humanidade através das civilizações, recebendo conotações distintas de acordo com os conceitos de saúde e bem estar e com a lógica da organização da sociedade em seu processo histórico de reprodução. Freitas (1) enfatiza que, a partir da Revolução Industrial, a população mundial mais que triplicou, a economia cresceu 20 vezes, o consumo de combustíveis fós- seis aumentou 30 vezes e a produção industrial cresceu 50 vezes. Paralelamente, ocorreu uma degradação do ambiente e da saúde. Esse autor considera que o crescimento e a expansão dos processos produtivos e de consumo contribuíram para que a ampliação da interdependência econômica fosse acompanhada de uma crescente interdependência ecológica (2); e que essas interdependências, associadas ao processo de globalização, contribuíram 
ativamente para ampliar as desigualdades socioespaciais. Ou seja, o processo atual de divisão internacional do trabalho, conhecido como globalização, além de ampliar as desigualdades econômicas e sociais, promove o acelerado consumo dos recursos naturais e a saturação dos ecossistemas através de rejeitos e contaminantes.

Segundo Soares et al. (3), nos últimos anos, a finalidade dos projetos de saneamento tem abandonado sua concepção sanitária clássica, recaindo em uma abordagem ambiental que visa a promover não só a saúde humana, mas também a conservação do meio físico e biótico. Segundo os mesmos autores, a avaliação ambiental dos efeitos dos sistemas de saneamento nas cidades consolidou-se como uma etapa importante do processo de planejamento no que se refere à formulação e seleção de alternativas e à elaboração e detalhamento dos projetos selecionados. Para ele, a avaliação da viabilidade ambiental, citando Pimentel e Cordeiro Netto (4), assume caráter de forte condicionante das alternativas a serem analisadas, ocorrendo, muitas vezes, a predominância dos critérios ambientais em relação, por exemplo, aos critérios econômicos.

Por outro lado, Heller (5) enfatiza que a ausência de instrumentos de planejamento relacionados à saúde pública é uma importante lacuna em programas governamentais no campo do saneamento no Brasil. Esse autor afirma que a compreensão das diversas facetas da relação do saneamento com a saúde pública é um pressuposto fundamental para a efetiva orientação das intervenções em saneamento, no sentido da otimização de sua eficácia. Considera ainda que a informação representa uma exigência para o posterior avanço dos setores de saneamento e de saúde, rumo à sua integração.

Dois estudos $(6,7)$ que consideram a questão das doenças infecciosas nas últimas décadas afirmam que as estratégias de controle sanitário, assim como o modelo tradicionalmente hegemônico da epidemiologia-baseado na análise dos fatores de risco individuais e dos fatores de vigilância epidemiológica e de fronteiras-, são inade- quados frente às doenças emergentes e re-emergentes que vêm causando enormes impactos sobre a saúde, a economia e os ecossistemas. Esses autores assinalam a necessidade de uma abordagem integrada entre as partes e o todo, entre o lugar e o seu contexto globalizado, devendo ser entendido o binômio saúde/doença como um processo coletivo, recuperando o "lugar" (definido como uma conjunção de fatores históricos, sociais e ambientais que produzem no espaço geográfico contextos particulares dos problemas de saúde) como espaço organizado para análise e intervenção. Enfatizam a necessidade de identificar, para situações específicas, as relações entre as condições de saúde e seus determinantes culturais, sociais e ambientais, dentro dos ecossistemas modificados pelas atividades econômicas, através de um enfoque interdisciplinar e interinstitucional.

McMichael (8) já destacou que os riscos emergentes para a saúde das populações humanas não se originam na contaminação do ambiente local e nem no esgotamento dos recursos materiais. Segundo o autor, o risco surge do rompimento do equilíbrio dos sistemas naturais da biosfera, devido à sobrecarga imposta à capacidade "metabólica" do planeta. McMichael considera que o impacto da humanidade sobre o planeta nos obriga a levar em consideração a relação entre os sistemas ecológicos e o ótimo funcionamento da espécie, sendo o ótimo funcionamento aproximado ao conceito de "boa saúde"; ou seja, a saúde da população humana, após sucessivas gerações, só pode ser mantida se as demandas sobre o ecossistema estiverem dentro de sua capacidade.

Outros $(6,7)$ consideram que, ao contrário da padronização dos métodos aplicados aos estudos epidemiológicos, a variedade de situações associadas com o binômio saúde/doença, como um processo coletivo, e os seus determinantes culturais, sociais e ambientais, dentro dos ecossistemas modificados pelo trabalho humano, exigem o reconhecimento da necessidade de múltiplas abordagens teóricas e metodológicas, onde o acesso à infor- mação torna-se imprescindível para o controle social, o planejamento e a segurança internacional. Esse enfoque enfatiza a necessidade do acesso à informação, sendo a sua sintetização em indicadores adequadamente desenvolvidos uma importante ferramenta para a gestão do território e o controle social.

A construção de indicadores deve ser precedida por um entendimento do fenômeno a ser estudado. A qualidade dos indicadores depende não só dos dados que são utilizados no seu cálculo, mas, principalmente, da compreensão teórica desse fenômeno. Samaja (9) considera que o indicador é uma operação prévia ao próprio enunciado da informação. Onde há uma informação (dado), há, obrigatoriamente: 1) uma referência a algum ente ou fato (unidade de análise); 2) um campo semântico (o âmbito de sentido), que constitui o fundo ou paradigma que define as alternativas possíveis de serem informadas (variável); 3) um estado particular desse campo semântico que se atualiza frente às alternativas possíveis (valor); e 4) o procedimento pelo qual se estabelece ou se adverte qual é essa alternativa que foi selecionada e atualizada entre todas as alternativas possíveis de que se dispõem (indicador).

Segundo Briggs (10), os principais objetivos do uso de indicadores socioambientais são detectar situações de risco relacionadas a problemas ambientais, monitorar tendências no ambiente e identificar riscos potenciais à saúde, monitorar tendências na saúde resultantes de exposições a fatores de risco, comparar condições ambientais e de saúde em diferentes áreas, permitindo a identificação de áreas prioritárias, e avaliar o impacto de políticas e intervenções sobre as condições de saúde e ambiente. Considera que o trabalho de seleção de indicadores parte da busca das variáveis e das fontes de informações que melhor representem essa relação.

O saneamento básico compreendido como um conjunto de ações de abastecimento de água, esgotamento sanitário e coleta de lixo é considerado um direito dos cidadãos e um item im- 
prescindível de qualidade de vida. A necessidade de fornecer água com quantidade e qualidade adequadas e, ao mesmo tempo, recolher e tratar os dejetos humanos, é conseqüência do processo de urbanização e do adensamento humano. Desde as primeiras intervenções de saneamento nas grandes cidades, no fim do século XIX, houve uma redução significativa em indicadores como a mortalidade infantil e a ocorrência de epidemias. A estratégia de universalização de técnicas e meios para a sua implementação, que pareceu inexorável no século XX, mostrouse inadequada ou insuficiente para suprir as necessidades de todos.

No Brasil, assim como na maior parte dos países da América Latina, a população urbana vem adquirindo acesso à água através da expansão precária, acima da capacidade das redes de abastecimento, sem que, por outro lado, sejam promovidos a coleta e o tratamento de esgotos e lixo. A combinação entre a universalização do acesso a redes de abastecimento de água e a crescente vulnerabilidade das fontes superficiais e subterrâneas de água pode, ao invés de proteger a população, magnificar os riscos à saúde, através da ampliação da população exposta a agentes químicos e biológicos.

Cada vez mais, o processo de urbanização e adensamento populacional produz riscos que são característicos de grandes centros urbanos, com mananciais e redes de distribuição de água vulneráveis. Há diversos relatos de surtos de doenças de veiculação hídrica transmitidos pelo sistema de distribuição de água $(11,12)$. A expansão desses sistemas, nesse caso, pode atuar também como meio de amplificação de riscos. A decadência dos serviços públicos de saneamento na antiga União Soviética tem promovido um aumento dos riscos associados à distribuição de água devido à precariedade dos sistemas (13). O sistema de abastecimento, nesse caso, funciona mais como um veículo de difusão de agentes infecciosos do que como fator de proteção das populações (12). Ressalta-se, também para o Brasil, a insuficiência dos indicadores hoje amplamente utilizados para a avaliação das condições de sa- neamento e saúde. Segundo Lee e Schwab (14), os principais problemas enfrentados hoje pelos sistemas de abastecimento de água no Terceiro Mundo são ligados à vulnerabilidade e intermitência desses sistemas, mais do que à sua cobertura. A intermitência do regime de abastecimento, por sua vez, permite a intrusão de agentes patogênicos através da água contaminada nas redes de distribuição (15).

Diante desse quadro, os problemas de saúde relacionados ao saneamento se diversificam e complicam, não podendo ser apreendidos por indicadores de cobertura de serviços, amplamente utilizados para o diagnóstico do setor (16). O principal objetivo deste trabalho é avaliar as tendências de pesquisas sobre a relação entre as condições de saneamento e a saúde, que se refletem na escolha das variáveis e do desenho dos estudos, de seus objetivos e do contexto em que foram gerados os indicadores. Para isso, foi realizada uma revisão da literatura. Procurou-se, também, apontar as principais variáveis utilizadas nos artigos e as doenças ou agravos usados como marcadores de efeito de condições ou fatores de risco ambientais.

\section{MÉTODOS}

Procurando identificar as diferentes concepções e usos de indicadores na compreensão das relações entre saneamento e saúde, foi organizado um levantamento bibliográfico com foco nas ações de abastecimento de água e esgotamento sanitário. Outros aspectos do saneamento, como a drenagem de águas pluviais e a coleta e tratamento de lixo urbano e industrial não foram considerados neste levantamento, devido à dificuldade de correlacionar esses fatores de risco ambiental com problemas de saúde cujos ciclos e mecanismos de transmissão ou disseminação são mais complexos e menos conhecidos.

O levantamento foi realizado através de pesquisa na Medline (acessada pelo endereço http://bases.bireme.br/cgibin/wxislind.exe/iah/online/?Isis Script $=i a h /$ iah . $x i s \& b a s e=M E D L I N E \&$ lang=). Também foram consultados ou- tros artigos nas bases SciELO e LILACS, além de textos publicados em livros de organizações relevantes como Organização Mundial da Saúde (OMS), Organização Pan-Americana da Saúde (OPAS), Fundação Instituto Oswaldo Cruz (FIOCRUZ), Instituto Brasileiro de Geografia e Estatística (IBGE) e ministérios brasileiros da saúde e das cidades $(1,5,9,10,16,17)$.

Inicialmente, foram pesquisados todos os artigos disponíveis na Medline para o período de 1966 a 2005. Foram utilizados os termos saúde, indicador e saneamento ("sanitati\$" e "health" e "indic\$"), sendo identificados 427 artigos. Para os termos saúde, indicador e água ("water" e "health" e "indic\$"), foram obtidos 2780 artigos.

Foi realizada uma nova delimitação desse universo, considerando-se apenas os artigos publicados no período de 10 anos (de 1995 a 2004) e utilizando os termos saneamento e água simultaneamente ("sanitati\$" e "health" e "indic $\$$ " $\mathrm{e}$ "water"). A partir dessa nova delimitação, 103 artigos foram identificados, sendo apenas 17 considerados pertinentes à análise proposta, centrada na relação entre saúde e saneamento através da utilização de indicadores de saúde (18-34). Nesses artigos, foram identificados o método epidemiológico, a variável de saúde e de saneamento utilizada nos indicadores, o país da publicação e o ano. Procuramos ainda estabelecer comparações com o trabalho de Heller (5), uma revisão bibliográfica que abrange um período de mais de 50 anos, imediatamente anterior ao que é enfocado, mais detalhadamente, neste trabalho. $\mathrm{O}$ autor analisa um universo de 256 estudos publicados entre 1929 e 1995. Foram consideradas as semelhanças e as diferenças significativas entre os diferentes períodos abordados (1929 a 1995 e 1995 a 2004).

\section{RESULTADOS}

As principais características dos 17 estudos considerados relevantes para a presente análise encontram-se na tabela 1.

Comparando os resultados do presente estudo com os obtidos, em um 
TABELA 1. Principais características dos artigos que correlacionam saneamento e saúde publicados entre 1995 e 2004

\begin{tabular}{|c|c|c|c|c|c|c|}
\hline Estudo & Método epidemiológico & Variável saúde & Variável saneamento $^{\mathrm{a}}$ & Resultado ${ }^{a, b}$ & País & Ano \\
\hline Bailey e Archer (19) & Inquéritos seqüenciais & Diarréia & AAG-QUAL e higiene & $\begin{array}{l}\text { Negativo para AAG- } \\
\text { QUAL e positivo } \\
\text { para higiene }\end{array}$ & África do Sul & 2004 \\
\hline Borchardt et al. (20) & Coorte & Diarréia & $\begin{array}{l}\text { Densidade de fossas } \\
\text { sépticas }\end{array}$ & Positivo & Estados Unidos & 2003 \\
\hline Cifuentes et al. (21) & Inquérito & Diarréia & $\begin{array}{l}\text { AAG-QUAL, ESG, } \\
\text { gosto da água }\end{array}$ & $\begin{array}{l}\text { Negativo para AAG- } \\
\text { QUAL e positivo } \\
\text { para ESG e gosto } \\
\text { da água }\end{array}$ & México & 2002 \\
\hline Cifuentes et al. (22) & Inquérito & Diarréia & $\begin{array}{l}\text { AAG-QUAL, } \\
\text { habitação, ESG }\end{array}$ & $\begin{array}{l}\text { Negativo para AAG- } \\
\text { QUAL e positivo } \\
\text { para habitação e } \\
\text { ESG }\end{array}$ & México & 2002 \\
\hline Carneiro et al. (23) & Inquérito & Ascaris lumbricoides & AAG & Positivo & Brasil & 2002 \\
\hline Vila et al. (29) & Ecológico & Bócio endêmico & AAG-QUAL iodo & Positivo & Espanha & 1999 \\
\hline Raina et al. (30) & Prospectivo, seguimento & Diarréia & $\begin{array}{l}\text { AAG-QUAL } \\
\text { E. coli }\end{array}$ & Positivo & Canadá & 1999 \\
\hline Gorter et al. (31) & Prospectivo, seguimento & Diarréia & Higiene & Positivo & Nicarágua & 1998 \\
\hline McCarthy et al. (32) & Inquérito & Diarréia & AAG-QUAL & Positivo & Suécia & 1998 \\
\hline $\begin{array}{l}\text { van Poppel e van der } \\
\text { Heijden (33) }\end{array}$ & Ecológico & Mortalidade infantil & AAG & Negativo & Holanda & 1997 \\
\hline Tang et al. (34) & Ecológico & $\begin{array}{l}\text { Diarréia, hepatite A, } \\
\text { febre tifóide }\end{array}$ & AAG-QUAL & Positivo & China & 1996 \\
\hline
\end{tabular}

a $\mathrm{AAG}$ = abastecimento de água; $\mathrm{AAG}-\mathrm{QUAL}$ = qualidade da água; $\mathrm{ESG}$ = esgotamento sanitário.

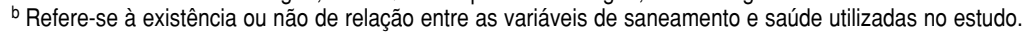

período anterior, por Heller (5), quanto ao número de artigos selecionados e a proporção de artigos por década, foi observada uma redução: 105 trabalhos foram selecionados por Heller na década de 1980 contra apenas 17 selecionados por nós para o período de 1995 a 2004. Quanto ao continente de origem das observações, enquanto quase $30 \%$ dos artigos revisados por Heller (5) eram provenientes da Ásia e $28 \%$ da África, neste estudo a maior participação foi da Europa, com $41,2 \%$ das publicações, seguida pelos Estados Unidos, com 35,3\%.

Em relação ao aspecto do saneamento avaliado nos artigos, o abastecimento de água foi abordado por $77,4 \%$ dos estudos no trabalho de Heller (5) e por $88,0 \%$ dos artigos no presente trabalho. Mesmo assim, a variável de saneamento empregada sofreu uma modificação importante, passando os estudos de qualidade da água a ser predominantes, aumentando de em torno de 20,0 para 59,0\%. Quanto ao indicador de saúde empregado, a diarréia foi utilizada em 10 dos 17 estudos selecionados neste trabalho (1995 a 2004), contra menos da metade dos artigos revisados por Heller (5). Em relação à faixa etária, o predomínio em todos os períodos foi de crianças entre 1 a 5 anos. Quanto à existência de associação entre as variáveis de saúde e as de saneamento, dos 17 artigos estudados por nós, apenas dois (12\%) não relataram alguma associação, enquanto Heller (5) encontrou, para a diarréia, 32\% de resultados negativos, ou seja, não havia associação.

O tipo de desenho epidemiológico predominante nos estudos analisados foi o ecológico $(41,2 \%)$, seguido pelos estudos seccionais ou inquéritos $(29,4 \%)$. Nas observações de Heller (5), os estudos ecológicos não ultrapassaram $10 \%$ dos artigos. Heller observou, inclusive, uma tendência de redução dos estudos ecológicos. Os dados atuais podem representar uma recuperação desse tipo de desenho para questões relativas ao saneamento e saúde. Observa-se, também, que os artigos com desenhos baseados em inquéritos utilizaram predominantemente a diarréia como indicador, enquanto que os artigos de desenho ecológico utilizaram uma diversidade maior de dados obtidos através de fontes secundárias. Nos artigos selecionados no período mais recente, observou-se uma maior preocupação em estabelecer relações entre a qualidade da água e doenças não transmissíveis, como o bócio endêmico e malformações congênitas $(27,29)$. Nos artigos revisados por Heller (5), predominaram as doenças transmissíveis de veiculação pela água.

\section{DISCUSSÃO}

A diferença entre o número de artigos selecionados neste artigo em re- 
lação ao de Heller (5) se deve, provavelmente, aos diferentes critérios de seleção e às estratégias de identificação utilizados pelos dois estudos. No entanto, a comparação entre os dois levantamentos permite identificar algumas tendências relativas aos métodos e aos resultados das pesquisas sobre saneamento e saúde. Em relação à maior associação entre as variáveis de saúde e as de saneamento observada neste estudo, é possível que avanços no desenho e nas ferramentas estatísticas possam ter contribuído para uma maior positividade.

Borchardt et al. (20), em um estudo do tipo caso-controle com populações rurais que residem perto de uma cidade, no Estado de Wisconsin, Estados Unidos, identificaram o risco atribuído à densidade de fossas sépticas em determinado espaço para diarréias por vírus e por bactérias. Esses autores encontraram um incremento de $8 \%$ nas diarréias virais para cada fossa séptica adicional para uma área de 640 acres, e um incremento de $22 \%$ para as diarréias bacterianas por fossa adicional para uma área de 40 acres. Os autores sugerem que esses sistemas de esgoto são uma importante fonte de contaminação do solo e da água, tendo seus efeitos multiplicados pela dificuldade de manutenção e idade das instalações. Um aspecto peculiar desse artigo é o de associar sistemas de saneamento e suas áreas de abrangência com o efeito da diarréia por determinadas etiologias. Mesmo com grandes limitações, o artigo levanta aspectos ecológicos e características do lugar onde ocorre a transmissão, alertando para a necessidade de novos estudos desses aspectos.

Cifuentes et al. (22) não encontraram associação entre a incidência de diarréia e a proximidade de poços de abastecimento de água contaminados com coliformes fecais. Esse artigo descreve um estudo transversal, que envolveu mais de 700 crianças menores de 5 anos e foi realizado em dois momentos, na estação seca e na chuvosa. As residências selecionadas estavam dentro de um raio de 500 metros dos cinco poços de abastecimento de água selecionados. Nessa região estava sendo implantado um programa de reutilização das águas servidas para a agricultura, e o estudo procurava averiguar possíveis riscos para a saúde ocasionados pela contaminação do lençol freático. Apesar de apresentar prevalências de diarréia de mais de $10 \%$ (10,7\% na estação seca e $11,8 \%$ na chuvosa), não foi possível identificar uma relação estatisticamente significativa com a proximidade do poço contaminado por coliformes fecais. Os autores questionaram a utilização dos índices de coliformes fecais como padrão de potabilidade, citando a detecção de cistos de Giardia intestinalis e oocistos de Cryptosporidium parvum na água efluente de estações de tratamento.

Outros artigos, embora não tenham sido incluídos na análise comparativa, merecem algumas considerações. Ferreira et al. (35) identificaram o saneamento básico como responsável pela redução de um quarto da prevalência das parasitoses observadas no período de análise. Os autores procuraram estimar a prevalência das parasitoses intestinais na infância com base nos resultados de dois inquéritos domiciliares realizados na Cidade de São Paulo, em 1984 e 1985 e 1995 e 1996; eles estabelecem a tendência secular dessas enfermidades e analisam a sua determinação social. Segundo os dados relatados, as condições de moradia, saneamento e acesso a serviços de saúde evoluíram favoravelmente entre os inquéritos. A freqüência de crianças vivendo em casas de alvenaria com menos do que duas pessoas por cômodo (incluindo cozinha e banheiro) aumentou de 71,4 para $80,9 \%$. A disponibilidade de redes de água e esgoto passou de 43,2 para $69,1 \%$. O acesso a serviços básicos de saúde (indiretamente identificado pela cobertura completa das vacinas injetáveis ministradas, como regra geral, no atendimento de rotina de unidades básicas de saúde) subiu de 76,8 para $94,0 \%$. A moradia, o saneamento e o acesso a serviços de saúde mostraramse independente e significativamente associados ao risco de ocorrência de helmintoses, mesmo após controle da renda familiar, da escolaridade materna e das demais variáveis de confundimento (idade da criança e ano do in- quérito). Reduções na prevalência de helmintoses, de 15,5, 11,6 e 8,9\%, correspondentes, respectivamente, a um quarto, um quinto e um sétimo do declínio efetivo observado no período, seriam esperadas devido às melhorias com relação à disponibilidade de saneamento, ao acesso a serviços de saúde e às condições de moradia.

Fehr et al. (36) abordaram questões sobre a privatização dos serviços de fornecimento de água e alguns efeitos na saúde, como o aumento de casos de diarréia decorrentes do corte de fornecimento para os inadimplentes, observado na Inglaterra. Os autores abordaram ainda a questão da qualidade da água na Alemanha e as dificuldades legais e econômicas de se implementar parâmetros ou de modificá-los no sentido de preservar a população da exposição a substâncias cancerígenas como o arsênio. Em outras palavras, os autores alertam para o fato de que necessidades como as de inclusão ou alteração de parâmetros de qualidade da água podem ser inexeqüíveis, do ponto de vista financeiro, para empresas privadas de prestação de serviço, podendo representar risco de exposição para a população. Trata-se de um aspecto muito pouco estudado, do ponto de vista dos impactos para a saúde.

Schwartz et al. (37), no Zimbábue, realizaram um inquérito enfocando a opinião do usuário sobre o sistema de abastecimento de água e os seus impactos na saúde. Os autores levantaram questões sobre o impacto da baixa qualidade da água e da irregularidades dos serviços na saúde da população, mesmo com altos índices de cobertura e instalações domiciliares.

McKay e Moeller (38) afirmam que o grande número de pessoas potencialmente suscetíveis e o risco de que pequenas alterações possam levar a efeitos na saúde da população em larga escala justificam a importância da qualidade da água em sistemas de abastecimento. Apontaram também que a poluição gerada por defensivos agrícolas e determinadas algas são fatores ainda pouco considerados, assim como as falhas de operação do sistema, para a garantia da qualidade da água de consumo da população. Vale a 
pena destacar os artigos de Auld et al. (39) e de Mygind et al. (40), que abordaram a questão da contaminação do sistema de abastecimento de água devido a fenômenos meteorológicos, como chuvas e inundações, chamando a atenção para as possíveis fragilidades do sistema e os impactos sazonais na saúde. Esses artigos demonstraram uma crescente preocupação no sentido de relacionar a saúde da população a aspectos ambientais mais gerais, como o regime de chuvas, as inundações e a qualidade da água de mananciais.

\section{CONCLUSÕES}

O presente artigo procurou complementar revisões anteriores com as observações de artigos publicados nos 10 anos seguintes. Pode-se destacar que aspectos relacionados ao ambiente se encontram em uma nova fase de valorização, onde as metodologias de estudos ecológicos com enfoques ecossistêmicos ganham consistência e mel- hores ferramentas, como os sistemas de informações geográficas (SIG) e técnicas de análise estatística como a regressão múltipla, entre outras (41).

Nos artigos aqui abordados, destacase a franca opção pelo uso da incidência de diarréias em crianças de 1 a 4 anos como a variável de saúde de escolha na relação com os aspectos do saneamento. $\mathrm{O}$ uso da diarréia como indicador prevalece por ser ela um sinal comum a diversas patologias, com uma diversidade de vias de transmissão e agentes etiológicos, para o qual os aspectos relacionados ao consumo de bens e de serviços ou condições socioeconômicas são muito relevantes.

Ainda restam muitas lacunas no entendimento e na valorização do saneamento e de sua particular importância nos grandes centros urbanos e metrópoles, onde a dependência dos sistemas de água e esgotamento sanitário é muito grande, não existindo, na maioria das vezes, fontes alternativas de abastecimento viáveis. A lógica dos serviços comerciais de abastecimento esbarra na lógica dos ecossistemas urbanos, onde a capacidade de pagamento, o suprimento e as necessidades não ocupam os mesmos espaços, não permitindo análises setoriais simplistas, que não considerem a diversidade da ocupação do espaço na distribuição diferenciada dos riscos ambientais envolvidos.

A necessidade de mecanismos de planejamento e indicadores de controle e avaliação dos serviços de saneamento e de seus impactos na população são amplamente reconhecidos. Porém, a construção de indicadores adequados e eficientes depende da compreensão teórica do fenômeno, da qualidade dos dados e dos paradigmas e da visão de mundo ou posicionamento ideológico diante das análises a realizar com esses indicadores. Destaca-se a necessidade de uma abordagem integrada em seus diversos níveis, do local ao global, para buscar identificar as relações entre as condições de saúde e seus determinantes dentro dos ecossistemas modificados pelo trabalho humano.

\section{REFERÊNCIAS}

1. Freitas CM. Subsídios para um debate sobre as inter-relações produção, consumo, saúde e meio ambiente. Em: Minayo MCS, org. Saúde e ambiente sustentável: estreitando nós. Rio de Janeiro: Fundação Instituto Oswaldo Cruz; 2002. Pp. 261-9.

2. MacNeill J, Winsemius P, Yuakushiji T. Para além da interdependência: a relação entre economia mundial e ecologia da terra. Rio de Janeiro: Jorge Zahar; 1992.

3. Soares SR, Bernardes RS, Netto OM. Relações entre saneamento, saúde pública e meio ambiente: elementos para formulação de um modelo de planejamento em saneamento. Cad Saude Publica. 2002;18(6):1713-24.

4. Pimentel CEB, Cordeiro Netto OM. Proposta metodológica de classificação e avaliação ambiental de projetos de saneamento. Brasília: IPEA; 1998.

5. Heller L. Saneamento e saúde. Brasília: OPAS; 1997.

6. Sabroza PC, Watner-Toews D. Doenças emergentes, sistemas locais e globalização. Cad Saude Publica. 2001;17(supl):4-5.

7. Walters-Toews D. An ecosystem approach to health and its applications to tropical an emerging diseases. Cad Saude Publica. 2001; 17(supl):7-36.

8. McMichael AJ. Planetary overload and human health: global environmental change and the health and survival of the human species. Cambridge: University Press; 1993.
9. Samaja J. Epistemología y metodología. Elementos para una teoría de la investigación científica. Buenos Aires: Eudeba; 1994.

10. Briggs D. Environmental health indicators: frameworks and methodologies [site da Internet]. Genebra: World Health Organization; 1999. Disponível em: http://whqlibdoc.who. int/hq/1999/WHO_SDE_OEH_99.10.pdf. Acessado em novembro de 2005.

11. Godoy P, Borrull C, Palà M, Caubet I, Bach P, Nuín $C$, et al. Brote de gastroenteritis por agua potable de suministro público. Gac Sanit. 2003;17(3):204-9.

12. Winston G, Lerman S, Goldberger S, Collins $\mathrm{M}$, Leventhal A. A tap water turbidity crisis in Tel Aviv, Israel, due to technical failure: toxicological and risk management issues. Int J Hyg Environ Health. 2003;206(3):193-200.

13. Semenza JC, Roberts L, Henderson A, Bogan J, Rubin $\mathrm{CH}$. Water distribution system and diarrheal disease transmission: a case study in Uzbekistan. Am J Trop Med Hyg. 1998;59(6): 941-6.

14. Lee EJ, Schwab KJ. Deficiencies in drinking water distribution systems in developing countries. J Water Health. 2005;3(2): 109-27.

15. LeChevallier MW, Gullick RW, Karim MR, Friedman M, Funk JE. The potential for health risks from intrusion of contaminants into the distribution system from pressure transients. J Water Health. 2003;1(1):3-14.
16. Barcellos C. Constituição de um sistema de indicadores socioambientais. Em: Minayo MCS, Miranda AC, orgs. Saúde e ambiente sustentável: estreitando nós. Rio de Janeiro: Fundação Instituto Oswaldo Cruz; 2002. Pp. 313-29.

17. Heller L, Moraes LRS, Monteiro TCN, Salles MJ, Almeida LM, Cancio J. Saneamento e saúde nos países em desenvolvimento. Rio de Janeiro: CC\&P Editores; 1997.

18. Krewski D, Balbus J, Butler-Jones D, Haas C, Isaac-Renton J, Roberts K, et al. Managing the microbiological risks of drinking water. J Toxicol Environ Health A. 2004;26;67(20-22): 1591-617.

19. Bailey IW, Archer L. The impact of the introduction of treated water on aspects of community health in a rural community in Kwazulu-Natal, South Africa. Water Sci Technol. 2004;50(1):105-10.

20. Borchardt MA, Chyou PH, DeVries EO, Belongia EA. Septic system density and infectious diarrhea in a defined population of children. Environ Health Perspect. 2003;111(5): 742-8.

21. Cifuentes E, Mazari-Hiriart M, Carneiro F, Bianchi F, Gonzalez D. The risk of enteric diseases in young children and environmental indicators in sentinel areas of Mexico City. Int J Environ Health Res. 2002;12(1):53-62.

22. Cifuentes E, Suarez L, Solano M, Santos R. Diarrheal diseases in children from a water reclamation site in Mexico city. Environ Health Perspect. 2002;110(10):A619-24. 
23. Carneiro FF, Cifuentes E, Tellez-Rojo MM, Romieu I. The risk of Ascaris lumbricoides infection in children as an environmental health indicator to guide preventive activities in Caparao and Alto Caparao, Brazil. Bull World Health Organ. 2002;80(1):40-6.

24. Gofti-Laroche L, Gratacap-Cavallier B, Genoulaz $\mathrm{O}$, Joret JC, Hartemann $\mathrm{P}$, Seigneurin $\mathrm{JM}$, et al. A new analytical tool to assess health risks associated with the virological quality of drinking water (EMIRA study). Water Sci Technol. 2001;43(12):39-48.

25. Gulis G. Life expectancy as an indicator of environmental health. Eur J Epidemiol. 2000; 16(2):161-5.

26. Gulis G, Kross BC. Drinking water, mortality, and life expectancy: an assessment of the eastwest mortality gap in Europe. Cent Eur J Public Health. 1999;7(4):191-6.

27. Magnus P, Jaakkola JI, Skrondal A, Alexander J, Becher G, Krogh T, et al. Water chlorination and birth defects. Epidemiology. 1999;10(5): 513-7.

28. Gutierrez G, Reyes H, Fernandez S, Perez L, Perez-Cuevas R, Guiscafre H. Impacto de los servicios de salud, el saniamento y la alfabetización en la mortalidad de menores de cinco años. Salud Publica Mex. 1999;41(5):368-75.

29. Vila L, Subirats E, Vila T, Margalef N, Vallescar $\mathrm{R}$, Leiva A. Estudio de bocio endémico en una población de los Pirineos (Cerdanya-Girona). An Med Intern (Madrid). 1999;16(7):338-44
30. Raina PS, Pollari FL, Teare GF, Goss MJ, Barry DA, Wilson JB. The relationship between $\mathrm{E}$. coli indicator bacteria in well-water and gastrointestinal illnesses in rural families. Can J Public Health. 1999;90(3):172-5.

31. Gorter AC, Sandiford P, Pauw J, Morales P, Perez RM, Alberts H. Hygiene behaviour in rural Nicaragua in relation to diarrhoea. Int J Epidemiol. 1998;27(6):1090-100.

32. McCarthy N, de Jong B, Ziese T, Sjolund R, Hjalt CA, Giesecke J. Epidemiological explanation of an outbreak of gastro-enteritis in Sweden in the absence of detailed microbiological information. Eur J Epidemiol. 1998;14(7): 711-8.

33. van Poppel F, van der Heijden C. The effects of water supply on infant and childhood mortality: a review of historical evidence. Health Transit Rev. 1997;7(2):113-48.

34. Tang M, Fan Y, Wang G. [Comprehensive cost-benefit evaluation for the improvement of rural water supply in Hunan province]. Zhonghua Yu Fang Yi Xue Za Zhi. 1996;30(1): 23-5.

35. Ferreira MU, Ferreira CS, Monteiro CA Tendência secular das parasitoses intestinais na infância na cidade de São Paulo (1984-1996). Rev Saude Publica 2000;34(6 Supl): 73-82.

36. Fehr R, Mekel O, Lacombe M, Wolf U. Towards health impact assessment of drinkingwater privatization: the example of water- borne carcinogens in North Rhine-Westphalia (Germany). Bull World Health Organ. 2003; 81(6):408-14.

37. Schwartz U, Siziya S, Tshimanga M, Barduagni P, Chauke TL. The impact of an inadequate municipal water system on the residents of Chinhoyi town, Zimbabwe. Cent Afr J Med. 1999;45(6):148-54.

38. McKay J, Moeller A. Is risk associated with drinking water in Australia of significant concern to justify mandatory regulation? Environ Manage. 2001;28(4):469-81.

39. Auld H, MacIver D, Klaassen J. Heavy rainfall and waterborne disease outbreaks: the Walkerton example. J Toxicol Environ Health A. 2004;(20-22)67:1879-87.

40. Mygind O, Laursen E, Rasmussen B, Ronne T. [Contamination of a water system with sewage]. Ugeskr Laeger. 1995;157(34):4676-9.

41. Nuckols JR, Ward MH, Jarup L. Using geographic information systems for exposure assessment in environmental epidemiology studies. Environ Health Perspect. 2004 Jun 112(9):1007-15

Manuscrito recebido em 16 de março de 2007. Aceito em 6 de junho de 2007

ABSTRACT Objective. To review the literature published from 1995-2004 on the relationship between sanitation and health and to identify the main variables analyzed and the diseases or injuries used as markers of effect or environmental health.

Old indicators for new problems: the relationship between sanitation and health

Key words
Method. A search of Medline, SciELO, and LILACS on "sanitation" and "health" and "indicator" and "water" produced 103 articles, 17 of which were considered relevant for the analysis. We identified the study design and the sanitation and health variables analyzed in each of the 17 studies.

Results. The most common study design was ecological and was employed in seven of the 17 studies. In most (10 studies), the health variable was diarrhea and sanitation variable was water quality. Fifteen studies reported a positive association between the health and sanitation variables.

Conclusions. There are still many gaps in our understanding of sanitation and its role, especially in large urban centers that are dependent on water and sewerage systems and often lack alternative water sources. Ecological studies based on secondary data and specific surveys have been shown to be a good option for analyses correlating sanitation and health.

Health status indicators, environmental monitoring, sanitation, environmental health, review literature. 